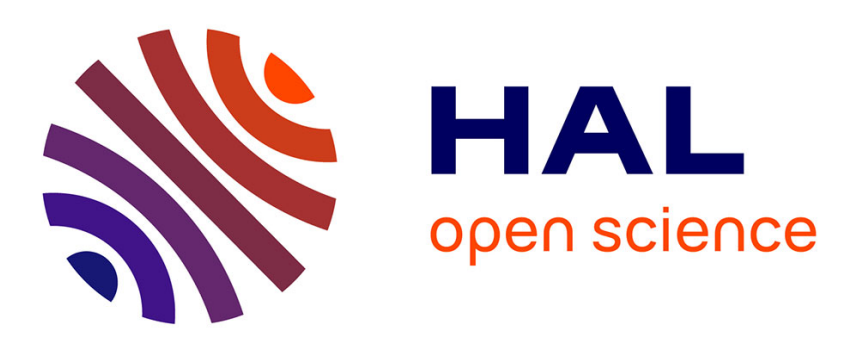

\title{
Implication des lipides dans la variabilité génétique des caractéristiques technologiques de la farine et du gluten de blé tendre
}

Bernard Laignelet, Catherine Cantin

\section{- To cite this version:}

Bernard Laignelet, Catherine Cantin. Implication des lipides dans la variabilité génétique des caractéristiques technologiques de la farine et du gluten de blé tendre. Agronomie, 1987, 7 (5), pp.345-351. hal-00885000

\section{HAL Id: hal-00885000 \\ https://hal.science/hal-00885000}

Submitted on 1 Jan 1987

HAL is a multi-disciplinary open access archive for the deposit and dissemination of scientific research documents, whether they are published or not. The documents may come from teaching and research institutions in France or abroad, or from public or private research centers.
L'archive ouverte pluridisciplinaire HAL, est destinée au dépôt et à la diffusion de documents scientifiques de niveau recherche, publiés ou non, émanant des établissements d'enseignement et de recherche français ou étrangers, des laboratoires publics ou privés. 


\title{
Implication des lipides dans la variabilité géné- tique des caractéristiques technologiques de la farine et du gluten de blé tendre
}

\author{
Bernard LAIGNELET \\ avec la collaboration technique de Catherine CANTIN
}

I.N.R.A., Laboratoire de Technologie des Céréales, Centre de Recherches de Montpellier, 9, place Viala, F 34060 Montpellier Cedex

Une étude a été entreprise pour rechercher les relations possibles entre les propriétés viscoélastiques du gluten de farines de blé tendre et la quantité et la répartition des lipides dans le gluten entier ou des fractions de ce gluten. Pour cela 23 échantillons, choisis au hasard parmi des lignées en fin de sélection cultivées dans le même lieu de culture en 1979 ont été analysés. Ils sont représentatifs d'une large gamme de volume de pain (450 à $900 \mathrm{ml}$ ). L'originalité de l'étude est de prendre en compte non pas tous les constituants de la farine mais seulement ceux qui participent au gluten. En moyenne, les protéines du gluten se partagent par moitié entre les fractions solubles et insolubles dans l'acide acétique $\mathrm{N} / 100$. Les lipides se partagent dans les mêmes fractions dans la proportion 1/3-2/3, et présentent une très forte variabilité. Au niveau technologique, il y a deux groupes de caractéristiques non reliés entre eux : d'une part le volume du pain et la quantité de gluten, d'autre part l'hydratation et la viscoélasticité de ce gluten.

Le volume du pain est corrélé fortement aux protéines et aux lipides participant au gluten, les coefficients de corrélation n'étant pas significativement différents entre eux. Plus il y a de gluten extractible, moins le gluten est riche en lipides surtout dans sa fraction insoluble. Si la teneur en protéines du gluten n'est pas reliée à sa viscoélasticité, on constate une différence significative entre les fractions solubles et insolubles : les premières ont un effet dépressif sur la viscoélasticité du gluten, à la différence des secondes. Par contre, les lipides qui se fixent dans le gluten sont corrélés hautement significativement à sa viscoélasticité, surtout ceux de la fraction insoluble.

Les besoins en recherches complémentaires et la possibilité d'un test de sélection du blé tendre sur sa valeur d'utilisation sont discutés.

Mots clés additionnels : Volume du pain, viscoélasticité du gluten, lipides neutres, glycolipides, phospholipides, gliadines, gluténines.

Implication of lipids in the genetical variation of technological characteristics of bread wheat flour and gluten.

A study of possible relations has been undertaken between the viscoelastic properties of bread wheat flour and the quantity and distribution of total gluten lipids and of fractionated gluten lipids. For this purpose, 23 random samples of breeding lines, grown at the same location in 1979, were analyzed. They covered a very large quality range (L. V. from 450 to $900 \mathrm{ml}$ ). The main originality of this work was to take into consideration not all the flour components but only the components implicated in gluten formation. Gluten proteins generally divided equally between fractions soluble and non-soluble in N/100 acetic acid. In the same fractions, lipids were present in 1/3-2/3 proportion, with a very high variation. From a technological point of view, two types of independent characteristics were found : on the one hand loaf volume and gluten quantity, on the other hand gluten hydration and viscoelacticity. Loaf volume was strongly correlated with gluten proteins and with gluten lipids, the correlation coefficients not being significantly different. The more the extractable gluten, the less the gluten was lipid-rich, mainly in the insoluble fraction. Overall there was no relation between gluten protein content and viscoelasticity but a significant difference appeared between fractions: the soluble fraction was correlated with viscoelasticity, while the insoluble one was not. In contrast, gluten lipids were highly significantly correlated with viscoelasticity, especially those in the insoluble fraction.

Future research possibilities and the potential of a breeding test based on lipids and proteins are discussed.

Additional key words : Loaf volume, gluten viscoelasticity, neutral lipids, glycolipids, phospholipids, gliadins, glutenins. 


\section{LISTE DES ABRÉVIATIONS UTILISÉES}

$\begin{array}{ll}\text { EMAG } & \begin{array}{l}\text { Ester méthylique d'acide gras } \\ \text { FSAA }\end{array} \\ \begin{array}{l}\text { Fraction du gluten soluble dans l'acide } \\ \text { acétique 0,01 N }\end{array} \\ \text { FIAA } & \begin{array}{l}\text { Fraction du gluten insoluble dans l'acide } \\ \text { acétique } 0,01 \mathrm{~N}\end{array} \\ \text { C } 16: 0 & \text { Acide palmitique } \\ \text { C } 17: 0 & \text { Acide heptadécanö̈que } \\ \text { C } 18: 0 & \text { Acide stéarique } \\ \text { C } 18: 1 & \text { Acide oléique } \\ \text { C } 18: 2 & \text { Acide linoléique } \\ \text { C } 18: 3 & \text { Acide linolénique }\end{array}$

\section{INTRODUCTION}

Les connaissances acquises ces dernières années sur les lipides des blés et leur importance en panification ont fait l'objet de plusieurs mises au point récentes (MORRISON, 1978; CHUNG \& POMERANZ, 1981; MAC RITCHIE, 1981 ; BERGER, 1982 ; BARNES, 1983 ; POMERANZ, 1985). Très schématiquement, les études sont orientées dans 2 directions : étude des lipides en tant que composants de la qualité des farines et étude des lipides polyinsaturés dans leurs implications dans des réactions d'oxydoréduction responsables de modifications de la valeur boulangère en particulier par agrégation covalente des sous-unités protéiques. Dans la première direction, il est généralement admis, à la suite de Chung \& Pomeranz (1981) \& POMERANZ (1985), que les protéines de la farine forment le réseau qui assure la structuration de la pâte et que les lipides renforcent et modifient cette structure pour changer les performances de la farine. Ainsi les liaisons des lipides avec les protéines du gluten semblent posséder une grande importance technologique. Dès 1969 cependant, GODON démontra l'existence des liaisons hydrogènes et hydrophobes qui assurent la structure des associations protéiques du gluten, les localisa et attribua un rôle aux lipides du gluten : les lipides se lient au gluten par des liaisons hydrophobes et forment des zones de jonction entre les protéines apolaires. Ces zones constituent alors des plans de glissement qui fournissent au gluten des propriétés plus plastiques. Dans la deuxième direction on sait maintenant que des lipides oxydés par l'activité des lipoxygénases endogènes ou exogènes ont tendance à se répartir au cours du pétrissage parmi les fractions les moins solubles des gluténines (LAIGNELET \& DUMAS, 1984) ce qui conduit à ne pas exclure l'agrégation covalente des protéines $\mathrm{P}$ par les lipides L sous la forme - P-00-L-00-P (CHEFTEl et al., 1985), la chaîne fournie pouvant se poursuivre. Ainsi, dans les 2 cas, on s'oriente vers l'étude de l'interaction des lipides avec les composants protéiques du gluten et de sa signification au niveau des propriétés physiques.

La présente étude porte sur la recherche de relations entre les propriétés viscoélastiques de gluten de farine de blé tendre extrait par lixiviation et la quantité et la répartition des lipides non amidon de ce gluten. Pour la mener à bien, il est nécessaire d'introduire une fluctuation des paramètres et 2 voies sont alors théoriquement possibles :
- étude par délipidation plus ou moins poussée des farines et des glutens ou étude par addition de lipides connus ; ces 2 premières voies font appel à l'utilisation de solvants des lipides dont l'effet, même à l'état de résidu, n'est pas négligeable sur les performances de la farine et peuvent conduire à des artéfacts ou des fausses interprétations (PONTE et al., 1967). De plus la première solution risque de dénaturer les agrégats lipoprotéiques d'une façon non contrôlée, surtout si la délipidation se fait à chaud, la deuxième comporte l'incertitude de savoir si les lipides ajoutés, le plus souvent du commerce, donc différents de ceux de blé, se placent aux mêmes sites d'action que ceux naturellement présents dans la farine ;

- étude par introduction d'une variabilité d'origine essentiellement génétique en minimisant les effets agroclimatiques et technologiques par l'utilisation d'échantillons cultivés la même année sur le même lieu de culture. En outre, en prenant des lignées en fin de sélection et non des variétés inscrites au catalogue, on peut élargir la variabilité technologique. C'est cette deuxième voie que nous avons retenue.

\section{MATÉRIEL ET MÉTHODES}

\section{A. Farines}

Les farines ont été extraites sur un moulin de laboratoire à partir de blés tendres en fin de sélection cultivés en 1979 à Versailles. Elles ont été obtenues en fonction de leur valeur boulangère par tirage au sort de 23 échantillons sur une pépinière de 120 environ comprenant de nombreuses hybridations, régulièrement répartis sur une gamme de volume de pain allant de 450 à $900 \mathrm{ml}$, de façon à avoir un échantillonnage très représentatif (méthode de micro-panification de BOURDET et al. (1972)).

\section{B. Extraction et fractionnement du gluten}

On a préparé un pâton en mélangeant $10 \mathrm{~g}$ de farine et $5 \mathrm{ml}$ d'eau désionisée. Après un repos de $20 \mathrm{mn}$, l'extraction du gluten a été effectuée par lixiviation manuelle sous l'eau désionisée.

Le gluten humide ainsi obtenu a été pesé et on en a mesuré les caractéristiques viscoélastiques. L'essai a été fait en double. On a ensuite rassemblé tous les glutens pour les lyophiliser et les peser à l'état sec.

Les teneurs en gluten humide et sec sont exprimées en p. $100 \mathrm{~m}$.s. (matière sèche) de farine. La capacité d'hydratation du gluten est égale à la teneur en eau du gluten en p. 100 de gluten humide.

Une partie du gluten lyophilisé broyé a été utilisée pour la détermination des teneurs en protéines et en lipides. Le reste a été fractionné de la façon suivante : $250 \mathrm{mg}$ de gluten lyophilisé sont mis en suspension dans $20 \mathrm{ml}$ d'acide acétique $0,01 \mathrm{~N}$. Après une agitation d'une nuit à $4{ }^{\circ} \mathrm{C}$, on centrifuge pendant $20 \mathrm{mn}$ à $3800 \mathrm{~g}$. Le culot de centrifugation est repris par $20 \mathrm{ml}$ d'acide acétique $0,01 \mathrm{~N}$, agité $1 \mathrm{~h}$ à $4{ }^{\circ} \mathrm{C}$ et centrifugé dans les mêmes conditions. Les surnageants sont rassemblés pour le dosage de la teneur en protéines, la teneur en protéines des fractions insolubles étant estimée par différence. Les fractions insolubles qui comprennent essentiellement les gluténines insolubles 
comme protéines, sont lyophilisées pour le dosage de la teneur en lipides, la teneur en lipides des fractions surnageantes étant estimée par différence.

\section{Mesures de la viscoélasticité du gluten humide}

Nous avons utilisé la méthode décrite par DAMIDAUX \& FEILLET (1978) et adaptée au gluten de blé tendre. Après fabrication d'une pastille de $1 \mathrm{~g}$ de gluten par thermoformage pendant $60 \mathrm{~s}$, les caractéristiques viscoélastiques sont déterminées avec un Viscoélastographe INRA-CHOPIN. Les réglages sont les suivants : contrainte $600 \mathrm{~g}$, durée d'application de la contrainte $40 \mathrm{~s}$, durée du retrait $20 \mathrm{~s}$. Les variables prises en considération sont :

- la fermeté égale à l'épaisseur de la pastille (en $\mathrm{mm}$ ) à la fin de l'application de la contrainte,

- la recouvrance élastique égale au gain d'épaisseur de la pastille $(\mathrm{en} \mathrm{mm}$ ) du fait du retrait de la contrainte.

Les déterminations sont faites en double sur chaque gluten extrait.

\section{Mesure de la teneur en protéines}

Les protéines des farines, du gluten sec et des fractions solubles dans l'acide acétique sont dosées à l'aide de la méthode Kjeldahl semi-automatique décrite par FEILLET (1976). Les résultats sont exprimés en p. $100 \mathrm{~m}$.s. en utilisant le coefficient de conversion 5,7 .

\section{E. Mesure de la teneur en lipides}

La teneur en lipides totaux a été déterminée sur les glutens et les fractions insolubles dans l'acide acétique lyophilisées par la méthode décrite par MORRISON et al. (1980). Après une hydrolyse acide des échantillons comportant un étalon interne d'acide heptadecanoïque C 17 : 0 , les acides gras résultants sont estérifiés par le méthanol en présence de $\mathrm{BF}_{3}$, purifiés par chromatographie sur couche mince de gel de silice, remis en solution dans l'éther diéthylique pour analyse par chromatographie en phase gazeuse. Les plaques de chromatographie sur couche mince sont développées avec du toluène, la détection se fait à l'aide de 2,7-dichlorofluorescéine. La colonne de chromatographie en phase gazeuse est en acier inoxydable $(2 \mathrm{~m} \times 2 \mathrm{~mm} \varnothing$ intérieur) remplie de Gas-Chrom P 100-120 mesh avec 15 p. 100 de EGSS-X travaillant à $185^{\circ} \mathrm{C}$. Le gaz vecteur est de l'azote au débit de $15 \mathrm{ml} / \mathrm{mn}$ et on utilise un détecteur à ionisation de flamme couplé avec un enregistreur intégrateur. Chaque solvant utilisé a été repurifié ou redistillé.

Les analyses sont faites en quadruple et les résultats sont exprimés en $\mathrm{mg} / 100 \mathrm{~g}$ m.s. d'esters méthyliques d'acide gras (EMAG). Il est possible d'estimer la teneur en lipides totaux en utilisant le coefficient multiplicateur de 1,20 (MORRISON et al., 1975).

\section{F. Mesure de la teneur en eau}

La teneur en eau des farines est déterminée sur $10 \mathrm{~g}$ à $130^{\circ} \mathrm{C}$ pendant $1 \mathrm{~h}$ avec l'étuve semi-automatique ventilée Brabender.

La teneur en eau des produits lyophilisés a été, après sondage, estimée être uniforme et égale à 3 p. 100 m.s.

\section{G. Analyses statistiques}

Seules des analyses statistiques paramétriques classiques ont été réalisées. Les comparaisons des coefficients de corrélation linéaire entre eux ont été réalisées par le test de Student après la transformation habituelle de $\mathrm{r}$ en $\mathrm{z}=$ Arg th $\mathrm{r}$.

\section{RÉSULTATS ET DISCUSSION}

\section{A. Variabilité des caractéristiques étudiées}

Le tableau 1 donne les valeurs moyennes et les coefficients de variation des caractéristiques étudiées. Les

TABLEAU

Valeur moyenne des paramètres utilisés dans l'étude sur la variabilité génétique de caractéristiques technologiques de farines de blé ( $n=23$ échantillons)

Mean values of parameters used in the study of genetical variation of technological characteristics of bread wheat flours $(n=23$ samples).

\begin{tabular}{|c|c|c|c|c|c|}
\hline & $\begin{array}{c}\text { Numéro } \\
\text { de variable }\end{array}$ & Unité & Moyenne & Ecart-type & $\begin{array}{l}\text { Coefficient } \\
\text { de variation }\end{array}$ \\
\hline Volume du pain & $(1)$ & $\mathrm{ml}$ & 599 & 132,8 & 22,2 \\
\hline Teneur en protéines de la farine & (2) & p. $100 \mathrm{MS}$ & 12,2 & 1,57 & 12,9 \\
\hline Teneur en protéines du gluten sec & (3) & p. $100 \mathrm{MS}$ & 79,6 & 2,72 & 3,4 \\
\hline Teneur en gluten humide de la farine & (4) & p. $100 \mathrm{MS}$ & 36,0 & 5,19 & 14,4 \\
\hline Teneur en gluten sec de la farine & (5) & p. $100 \mathrm{MS}$ & 12,9 & 1,83 & 14,2 \\
\hline Hydratation du gluten & (6) & p. 100 gluten humide & 64,1 & 2,40 & 3,7 \\
\hline Fermeté & (7) & $\mathrm{mm}$ & 1,91 & 0,244 & 12,8 \\
\hline Recouvrance élastique & $(8)$ & $\mathrm{mm}$ & 1,31 & 0,385 & 29,4 \\
\hline Teneur en lipides du gluten (EMAG) & (9) & p. 100 gluten sec & 5,40 & 0,836 & 15,5 \\
\hline Teneur en lipides FSAA (EMAG) & $(10)$ & p. 100 protéines FSAA & 3,82 & 1,355 & 35,5 \\
\hline Teneur en lipides FIAA (EMAG) & $(11)$ & p. 100 protéines FIAA & 6,88 & 0,950 & 13,8 \\
\hline Teneur en lipides du gluten (EMAG) & $(12)$ & p. 100 MS farine & 0,55 & 0,088 & 16,0 \\
\hline Teneur en lipides FSAA (EMAG) & (13) & p. 100 MS farine & 0,19 & 0,074 & 38,9 \\
\hline Teneur en lipides FIAA (EMAG) & (14) & p. 100 MS farine & 0,36 & 0,053 & 14,7 \\
\hline Teneur en protéines du gluten & $(15)$ & p. 100 MS farine & 10,2 & 1,66 & 16,3 \\
\hline Teneur en protéines FSAA & $(16)$ & p. 100 MS farine & 4,8 & 0,89 & 18,5 \\
\hline Teneur en protéines FIAA & $(17)$ & p. 100 MS farine & 5,3 & 1,13 & 21,3 \\
\hline
\end{tabular}


teneurs en protéines, en gluten humide et en gluten sec possèdent une variabilité du même ordre de grandeur $(\mathrm{CV}=13-15$ p. 100) alors que la capacité d'hydratation du gluten semble plus stable. Parmi les caractéristiques viscoélastiques du gluten, c'est la recouvrance qui présente la plus grande plage de variation $(\mathrm{CV}=$ 29 p. 100). La quantité de protéines de la farine qui participent à la formation du gluten est assez variable $(\mathrm{CV}=16 \mathrm{p} .100)$ ainsi que la répartition de ces protóines entre les fractions solubles et insolubles dans l'acide acétique $(\mathrm{CV}=18-21 \mathrm{p}$. 100), même si en moyenne elles se partagent par moitié entre chacune des fractions. On notera la variabilité particulièrement élevée des teneurs en lipides. En effet, le coefficient de variation peut avoisiner les 39 p. 100.

Les teneurs en lipides du gluten et de ses fractions ont 2 modes d'expression : soit elles sont calculées en pourcentage des protéines du gluten ou de la fraction considérée, soit elles sont rapportées à la farine. La première expression est justifiée par le fait que le gluten natif contient des quantités non négligeables de glucides surtout dans sa fraction gluténine où il atteint 17 p. 100 (MAC MASTER \& BUSHUK, 1983) et dont on ignore la variabilité.

Le gluten comprend en moyenne 5,4 p. 100 m.s. d'EMAG soit 6,5 p. 100 en lipides totaux avec un intervalle de variation de \pm 2 p. 100 pour $P=5 \mathrm{p} .100$. La fraction insoluble est plus riche que la fraction soluble dans l'acide acétique en lipides totaux $-8,3$ p. 100 et 4,6 p. 100 en moyenne respectivement - mais l'intervalle de variation le plus grand $( \pm 3,4$ p. 100 pour $P=5$ p. 100) est observé dans la fraction soluble. La répartition des lipides entre les fractions solubles et insolubles est en moyenne 1/3$2 / 3$. En se basant sur une teneur moyenne des farines de $1330 \mathrm{mg}$ EMAG/100 $\mathrm{g}$ de lipides non-amidon (BERGER, 1983), on peut calculer que 40 p. 100 environ des lipides de la farine participent au gluten, le reste étant entraîné avec l'amidon et les protéines solubles au cours de la lixiviation. Ce chiffre est très inférieur à celui publié par OLCOTT \& MECHAM (1947) selon lesquels 80 p. 100 des lipides de la farine se retrouvent dans la fraction gluténine et 5 p. 100 dans la fraction gliadine avec une variation induite par les conditions de fractionnement. Il est par contre supérieur à celui obtenu par NGUYEN-BREM et al.
(1983) qui ne retrouvent que 24 p. 100 de lipides de la farine dans le gluten après un léger pétrissage.

L'examen du tableau 2 sur la composition en acides gras des lipides du gluten et de ses fractions révèle des différences sensibles intéressant surtout les acides palmitique et linolénique. Ces différences doivent traduire des différences de classes lipidiques.

\section{B. Relations entre les caractéristiques technologiques de la farine et du gluten}

Il ressort du tableau 3 que les caractéristiques technologiques de la farine et du gluten forment 2 groupes : d'une part le volume du pain, la teneur en protéines du gluten, les teneurs de la farine en gluten humide et sec, d'autre part la capacité d'hydratation et les propriétés viscoélastiques du gluten (fermeté et recouvrance élastique). Entre ces 2 groupes il n'existe pas de coefficients de corrélation qui soient significativement différents de 0 , à l'exception de la teneur en gluten humide de la farine corrélée négativement avec les propriétés viscoélastiques. Le fait que l'on ne l'observe pas avec la teneur en gluten $\mathrm{sec}$, suggère qu'une capacité d'hydratation élevée se traduit par un effet de dilution défavorable des composants biochimiques responsables des propriétés viscoélastiques.

Pratiquement toutes les variables du premier groupe sont positivement corrélées entre elles d'une façon hautement significative et ceci n'est pas a priori surprenant car il s'agit de variables plus ou moins redondantes car toutes dérivées ou étroitement corrélées à la teneur en protéines de la farine (tabl. 4) qui est également fortement reliée au volume du pain.

La capacité d'hydratation et la viscoélasticité du gluten sont également très reliées entre elles : un gluten ferme a une forte recouvrance élastique et une faible capacité d'hydratation. Cependant, cette dernière caractéristique n'explique pas - au sens statistique du terme - à elle seule les propriétés viscoélastiques du gluten et le coefficient de corrélation linéaire $+0,911$ entre fermeté et recouvrance élastique ne signifie pas que ces deux variables soient redondantes. En effet, la corrélation partielle pour une hydratation fixée devient $+0,720$, significativement différente de $+0,911$ pour $\mathrm{P}=0,05$. Autrement dit les causes de la fermeté et de la recouvrance élastique, autres que

TABLEAU 2

Composition en acides gras des lipides du gluten et de ses fractions. Fatty acid composition of the lipids of gluten and its fractions.

\begin{tabular}{|c|c|c|c|c|c|c|}
\hline Acide gras & C $16: 0$ & C $18: 0$ & C $18: 1$ & C $18: 2$ & C $18: 3$ & TOTAL \\
\hline \multicolumn{7}{|l|}{ Gluten total } \\
\hline Moyenne & 27,1 & 1,1 & 8,9 & 60,2 & 2,7 & 100 \\
\hline Ecart-type & 1,77 & 0,23 & 1,69 & 2,76 & 0,46 & \\
\hline Coefficient de variation & 6,5 & 20,9 & 19,0 & 4,6 & 17,0 & \\
\hline \multicolumn{7}{|l|}{ Fraction SAA } \\
\hline Moyenne $\left({ }^{*}\right)$ & 19,4 & 0.3 & 7,0 & 68,7 & 4,6 & 100 \\
\hline \multicolumn{7}{|l|}{ Fraction IAA } \\
\hline Moyenne & 31,1 & 1,5 & 9,9 & 55,8 & 1,7 & 100 \\
\hline Ecart-type & 3,51 & 0.57 & 2,13 & 4,96 & 0,28 & \\
\hline Coefficient de variation & 11,3 & 38,0 & 21,5 & 8,9 & 16,5 & \\
\hline
\end{tabular}

(*) Moyenne calculée à partir des moyennes concernant le gluten total et la fraction IAA. 
TABLEAU 3

Corrélations linéaires entre les caractéristiques technologiques $(n=23$ échantillons). Linear correlations between technological characteristics $(n=23$ samples).

\begin{tabular}{|c|c|c|c|c|c|c|c|c|}
\hline & & $\begin{array}{l}\text { Volume du } \\
\text { pain } \\
\text { (1) }\end{array}$ & $\begin{array}{l}\text { Protéines du } \\
\text { gluten } \\
\text { (3) }\end{array}$ & $\begin{array}{c}\text { Gluten } \\
\text { humide } \\
\text { (4) }\end{array}$ & $\begin{array}{c}\text { Gluten } \\
\text { see } \\
(5)\end{array}$ & $\begin{array}{l}\text { Hydratation } \\
\text { du gluten } \\
\text { (6) }\end{array}$ & $\begin{array}{c}\text { Fermeté du } \\
\text { gluten } \\
\text { (7) }\end{array}$ & $\begin{array}{c}\text { Recouvrance } \\
\text { élastique du } \\
\text { gluten } \\
\text { (8) }\end{array}$ \\
\hline Volume du pain & (1) & 1 & & & & & & \\
\hline $\begin{array}{l}\text { Teneur en protéines } \\
\text { du gluten }\end{array}$ & (3) & 0,183 & 1 & & & & & \\
\hline $\begin{array}{l}\text { Teneur en gluten } \\
\text { humide }\end{array}$ & (4) & $0,609^{* *}$ & $0,552^{* *}$ & 1 & & & & \\
\hline Teneur en gluten sec & (5) & $0,773^{* *}$ & $0,608^{* *}$ & $0,892^{* *}$ & 1 & & & \\
\hline Hydratation du gluten & (6) & $-0,309$ & $-0,050$ & 0,292 & $-0,167$ & 1 & & \\
\hline Fermeté & (7) & $-0,225$ & $-0,065$ & $-0,424^{*}$ & $-0,012$ & $-0,897^{* *}$ & 1 & \\
\hline Recouvrance élastique & (8) & $-0,180$ & $-0,237$ & $-0,443^{*}$ & $-0,077$ & $-0,805^{* *}$ & $0,911^{* *}$ & 1 \\
\hline
\end{tabular}

$\left({ }^{*}\right): \mathrm{P}=0,05 ;\left({ }^{* *}\right): \mathrm{P}=0,01$.

l'effet de dilution évoqué plus haut, ne sont que partiellement communes $\left(\mathbf{R}^{2}=0,518\right)$, même si elles agissent dans le même sens. Ce fait mérite d'être souligné, même si à l'heure actuelle on n'en possède pas d'explication satisfaisante sur le plan biochimique.

\section{Relations entre les caractéristiques du gluten et la composition de la farine (tabl. 4)}

Au niveau des caractéristiques technologiques, nous retrouvons les 2 groupes mentionnés plus haut. Ainsi il existe de fortes corrélations positives hautement significativement différentes de 0 entre la teneur en protéines de la farine et le volume du pain, la teneur en gluten humide et en gluten sec, ce qui est en accord avec toutes les données de la littérature récemment revue par FINNEY (1985). Par contre, par principe même de la méthode de mesure, il n'est pas étonnant de constater que la teneur en protéines de la farine n'est pas reliée aux mesures au Viscoélastographe. La teneur en protéines du gluten sec n'est corrélée à aucune des variables étudiées dans le tableau 4.
Le volume du pain est corrélé positivement et d'une manière hautement significative à la quantité de protéines de la farine qui participent à la formation du gluten $\left(+0,812^{* *}\right)$ et à la quantité de lipides également retrouvés dans le gluten $\left(+0,643^{* *}\right)$, ces 2 coefficients de corrélation linéaire n'étant pas significativement différents entre eux $(t=1,12)$. Autrement dit en cas de relation causale, on n'a pas montré au sens statistique que les protéines ont un pouvoir explicatif plus important que les lipides ; même si on peut observer une faible corrélation (tabl. 5) entre ces 2 variables $\left(+0,420^{*}\right)$, la corrélation partielle entre le volume du pain et les protéines incorporées dans le gluten à teneur en lipides constante est de $+0,779 * *$ et celle entre volume du pain et lipides incorporés dans le gluten à teneur en protéines constante est de $0,571^{* *}$. Alors que l'étude des lipides totaux de la farine en relation avec le volume du pain donne des résultats divergents ou contradictoires dans la littérature (BARNES, 1983), la prise en considération des lipides qui se fixent dans le réseau glutineux amène à des coefficients de corrélation qui ne sont pas significati-

TABLEAU 4

Coefficients de corrélation linéaire entre les caractéristiques technologiques et la composition biochimique de la farine et du gluten $(n=23$ échantillons).

Linear correlation coefficients between technological characteristics and biochemical composition of flour and gluten ( $n=23$ samples).

Protéines de la farine Protéines gluten/farine Protéines FSAA/farine Protéines FIAA/farine t[16-17]

Lipides gluten/farine Lipides FSAA/farine Lipides FIAA/farine t[13-14]

Lipides gluten/gluten Lipides FSAA/protéines Lipides FIAA/protéines t[10-11]

\begin{tabular}{|c|c|c|c|c|c|c|c|}
\hline & $\begin{array}{c}\text { Volume du } \\
\text { pain } \\
\text { (1) }\end{array}$ & $\begin{array}{l}\text { Protéines du } \\
\text { gluten } \\
\text { (3) }\end{array}$ & $\begin{array}{c}\text { Gluten } \\
\text { humide } \\
\text { (4) }\end{array}$ & $\begin{array}{c}\text { Gluten } \\
\text { sec } \\
(5)\end{array}$ & $\begin{array}{c}\text { Hydratation } \\
\text { du gluten } \\
\text { (6) }\end{array}$ & $\begin{array}{l}\text { Fermeté du } \\
\text { gluten } \\
\text { (7) }\end{array}$ & $\begin{array}{l}\text { Recouvrance } \\
\text { élastique du } \\
\text { gluten } \\
\text { (8) }\end{array}$ \\
\hline (2) & $0,790^{* *}$ & 0,152 & $0,887 * *$ & $0,966^{* *}$ & $-0,103$ & $-0,028$ & $-0,065$ \\
\hline (15) & $0,812^{* *}$ & 0,258 & $0,880^{* *}$ & $0,964 * *$ & $-0,103$ & $-0,042$ & $-0,126$ \\
\hline (16) & $0,534^{* *}$ & 0,174 & $0,872 * *$ & $0,746^{* *}$ & $+0,318$ & $-0,403$ & $-0,498^{*}$ \\
\hline \multirow[t]{2}{*}{ (17) } & $0,773^{* * *}$ & 0,243 & $0,608^{* *}$ & $0,830^{* *}$ & $-0,401$ & 0,254 & $-0,205$ \\
\hline & 1,37 & 0,23 & 1,45 & 0,71 & $2,35^{* *}$ & $2,17^{*}$ & $2,39^{*}$ \\
\hline (12) & $0,643^{* * *}$ & 0,028 & 0,149 & $0,473^{*}$ & $-0,681^{* *}$ & $0,557^{* *}$ & $0,453^{*}$ \\
\hline (13) & $0,501^{*}$ & $-0,081$ & 0,223 & 0,346 & $-0,255$ & 0,197 & 0,103 \\
\hline \multirow[t]{2}{*}{ (14) } & 0,367 & 0,161 & $-0,064$ & 0,300 & $-0,772 * *$ & $0,681^{* *}$ & $0,607 * *$ \\
\hline & 0,52 & 0,77 & 0,89 & 0,13 & $2,42^{*}$ & $2,00^{*}$ & $1,90^{*}$ \\
\hline (9) & $-0,014$ & 0,020 & $-0,651^{* *}$ & $-0,412^{*}$ & $-0,559 * *$ & $0,614 * *$ & $0,533^{* *}$ \\
\hline (10) & 0,328 & $-0,148$ & 0,171 & 0,014 & $-0,409^{*}$ & 0,387 & 0,324 \\
\hline \multirow[t]{2}{*}{ (11) } & $-0,613^{* *}$ & $-0,105$ & $-0,840^{* *}$ & $-0,785^{* *}$ & $-0,168$ & 0,282 & 0,264 \\
\hline & $3,44^{* *}$ & 0,14 & $3,32 * *$ & $3,39^{* *}$ & 0,84 & 0,32 & 0,17 \\
\hline
\end{tabular}

$\left(^{*}\right): P=0,05 ;\left({ }^{* *}\right): P+0,01$. 
TABLEAU 5

Coefficients de corrélation linéaire entre les protéines et les lipides du gluten de la farine ( $n=23$ échantillons).

Linear correlation coefficients between the proteins and lipids of gluten from flour ( $n=23$ samples).

\begin{tabular}{|c|c|c|c|c|c|}
\hline & & $\begin{array}{c}\text { Protéines } \\
\text { de la } \\
\text { farine } \\
(2)\end{array}$ & $\begin{array}{l}\text { Protéines } \\
\text { gluten/ } \\
\text { farine } \\
(15)\end{array}$ & $\begin{array}{c}\text { Protéines } \\
\text { FSAA } \\
\text { farine } \\
\text { (16) }\end{array}$ & $\begin{array}{c}\text { Protéines } \\
\text { FIAA/ } \\
\text { farine } \\
(1))\end{array}$ \\
\hline $\begin{array}{l}\text { Lipides } \\
\text { gluten/farine } \\
\text { Lipides }\end{array}$ & (12) & 0,389 & $0,420^{*}$ & 0,170 & $0,487^{*}$ \\
\hline $\begin{array}{l}\text { FSAA/farine } \\
\text { Lipides }\end{array}$ & (13) & 0,312 & 0,303 & $0,402 *$ & 0,130 \\
\hline FIAA/farine & (14) & 0,210 & 0,272 & $-0,280$ & $0,619^{* *}$ \\
\hline
\end{tabular}

$(*): \mathrm{P}=0,05 ;\left({ }^{* *}\right): \mathrm{P}=0,01$

vement différents de ceux que l'on a avec les teneurs en protéines. Ces éléments vont dans le sens de synergie entre protéines et lipides telle que la conçoivent Chung \& Pomeranz (1981) \& Pomeranz (1985). La distinction entre fractions solubles et fractions insolubles dans l'acide acétique n'apporte pas d'informations nouvelles à ce niveau, les coefficients de corrélation linéaire n'étant pas significativement différents entre eux ni avec ceux qui concernent le gluten entier. La teneur en lipides du gluten ne semble apparemment pas jouer un rôle dans le volume du pain. Ainsi cette dernière caractéristique est favorablement dépendante de la part des lipides qui vont intervenir dans le gluten, et en première analyse que ce gluten contienne 4 ou 7 p. 100 de lipides semble indifférent. Or au cours du fractionnement, on voit apparaître des coefficients de corrélation de signes opposés dont les effets conjugués s'annulent mais hautement significativement différents entre eux $\left(t=3,34^{* *}\right)$. Ainsi, ramenée à la teneur en protéines de la fraction, la quantité de lipides qui interagissent avec ces gluténines insolubles est contraire à l'obtention d'un pain à volume élevé. Dans la fraction protéique soluble on observe le phénomène inverse, probablement prédominant compte tenu du signe et de la valeur du coefficient de corrélation linéaire entre le volume du pain et la quantité de lipides qui se fixent dans le gluten.

La teneur en protéines du gluten sec n'est pas corrélée avec l'une des caractéristiques biochimiques étudiées. Il n'en est pas de même des teneurs en gluten humide et en gluten sec, qui suivent une évolution parallèle. Il est ainsi normal que la teneur en gluten varie comme la teneur en protéines de la farine (cette détermination était d'ailleurs autrefois une méthode indirecte de mesure de la teneur en protéines de la farine) et que ces corrélations linéaires restent élevées pour les fractions solubles et insolubles dans l'acide acétique. L'extraction du gluten entraînant une partie des lipides liés aux protéines, on observe ainsi une corrélation significative et positive entre la quantité de lipides de la farine qui se fixent dans le gluten et la quantité de gluten extrait. Néanmoins, un facteur important mérite d'être noté : plus la quantité de gluten extrait manuellement est élevée, moins celui-ci est riche en lipides notamment dans le cas de la fraction insoluble. Ceci est confirmé par le fait que si la quantité de lipides liés au gluten évolue comme la quantité de gluten, plus la fraction gluténine insoluble est riche en lipides, moins le gluten est extractible, cette opposition étant hautement significative. Donc dans tous les cas le gluten contient des lipides, mais la quantité de lipides de gluténines insolubles semble être une gêne de l'extractibilité du gluten (à la différence de la fraction soluble dans l'acide acétique). Elle semble s'opposer à la formation du réseau glutineux probablement par le biais d'interactions protéines-lipides limitant les interactions directes entre les sous-unités protéiques.

Pour ce qui concerne les propriétés viscoélastiques du gluten, la teneur en protéines de la farine ainsi que celles qui participent au gluten semblent $a$ priori ne jouer aucun rôle. Il s'agit en fait du résultat de 2 phénomènes antagonistes: les teneurs en protéines des fractions solubles et insolubles dans l'acide acétique jouent un rôle opposé et significativement différent et qui ensembles annulent leurs effets : les fractions solubles évoluent d'une manière défavorable à une fermeté et une recouvrance élastique élevée, à la différence des fractions insolubles. Par contre, la quantité de lipides de la farine qui se fixent dans le gluten, ou de lipides du gluten est corrélée positivement et hautement significativement aux caractères viscoélastiques. En cas de relation fonctionnelle ceci est également vrai pour les lipides de la farine qui vont se fixer dans la fraction insoluble alors que les interactions entre lipides et fractions solubles protéiques n'auraient qu'un rôle mineur.

\section{DISCUSSION GÉNÉRALE ET CONCLUSION}

Les diverses observations rapportées plus haut montrent que sur une large gamme de qualité de farines de blé tendre, il existe un certain nombre de corrélations linéaires entre les lipides de la farine qui vont se fixer dans le gluten et des propriétés technologiques comme le volume du pain de cette dernière ou la quantité de gluten. Le tableau 5 confirme entre autres que le gluten possède une structure de complexe lipoprotéique et que l'étude qualitative et quantitative des lipides présents est nécessaire, que ces derniers soient sous forme oxydée - donc plus réactive avec les protéines - ou non. L'originalité essentielle de ce travail est de prendre en considération non pas les lipides totaux, mais ceux qui participent réellement au réseau glutineux. Leur étude fine reste cependant à faire. De nouveaux outils d'analyse biochimique des classes lipidiques d'une façon plus précise et plus fine que la quantification globale des lipides neutres, phospholipidiques ou glycolipides ou des lipides libres et liés doivent être mis en œuvre à la fois dans le but de comprendre les résultats obtenus par les biophysiciens et les biochimistes impliqués dans des travaux sur les agrégats.

Du point de vue du sélectionneur, la question qui se pose est de savoir si les lipides peuvent générer un test de sélection pour la valeur boulangère pour les blés français, à l'instar de ce qui se pratique déjà aux Etats-Unis à la Kansas State University (CHUNG, communication personnelle, 1982). Par analogie avec les protéines, on n'a pas envisagé de test de sélection qualitative par la détermination de la teneur en protéines du blé ou de la farine mais par la détermination par 
exemple du rapport gliadine/gluténine, de l'insoluble acétique, de la solubilité par les savons, du gel protéique, etc... c'est-à-dire par une quantification d'une classe protéique particulière faite dans des conditions très spécifiques. Il en est de même pour les lipides totaux de la farine dont on n'a jamais pu établir de corrélations linéaires claires avec des critères qualitatifs. Mais la situation est différente si l'on prend, comme dans ce travail, la teneur en lipides qui se fixent dans le gluten ou celles de fractions de ce gluten. Le rôle technologique de ces lipides commence à être connu. Ainsi ils participent à des phénomènes de lipolyse et d'oxydation soit en cours de la maturation de la farine, soit au pétrissage (GALliard, 1986a et $b$ ) ce qui se traduit entre autre par un blanchissement de la pâte et un renforcement des propriétés du gluten (OHTA et al., 1983). Les lipides oxydés ont tendance au cours du pétrissage à se fixer dans les fractions les plus insolubles (LAIGNELET \& DUMAS, 1984). Enfin, les lipides interviennent dans des phénomènes d'agrégation des protéines, des gliadines notamment (BEKES et al., 1983 ; MAC MASTER \& BuSHuK, 1983 ; POMERANZ, 1985) et on a isolé la ligoline qui fixe spécifiquement au cours du pétrissage des triglycérides et qui appartient à la classe des gluténines à faible poids moléculaire (FrazIER et al., 1981). Cependant, bien des points restent obscurs, en particulier les rôles relatifs des enzymes hydrolytiques et lipoxygénasiques et des divers lipides dont les effets, comme nous l'avons vu sont différents et/ou opposés. Il s'agit donc de rechercher ainsi un test simple, reproductible, rapide, peu onéreux, et ne nécessitant qu'une quantité limitée de produits. Ceci à l'heure actuelle n'est pas irréaliste compte tenu du développement rapide des techniques d'analyses des lipides. Un tel test pourrait venir en complément des tests existants, et non forcément, comme BUSHUK (comm. pers., 1983) le prétend au vu des corrélations linéaires qu'il obtient avec les critères de qualité et qui sont toutes supérieures à 0,95 , en substitution des tests existants.

Reçu le 7 avril 1986 Accepté le 27 janvier 1987

\section{REMERCIEMENTS}

L'auteur remercie Monsieur Jean-Claude Autran, responsable de l'Unité de Recherche "Biochimie Biologie Moléculaire», ainsi que le Prolesseur Paul Mazliak pour leurs critiques fructueuses et leurs conseils judicieux au cours de la rédaction de cet article.

\section{RÉFÉRENCES BIBLIOGRAPHIQUES}

Barnes P. J. (ed.), 1983. Lipids in Cereal Technology, 1 vol. Academic Press, Londres, $425 \mathrm{p}$.

Bekes F., Zawistowska U., Bushuk W., 1983. Protein-lipid complexes in the gliadin fraction. Cereal Chem., 60, 371-378.

Berger M., 1982. Les lipides du blé tendre. 1 - Etat actuel de nos connaissances. Sci. Aliments, 2, 411-450.

Berger M., 1983. Les lipides du blé tendre. 2 - Composition lipidique des farines de moutures d'essai de huit variétés françaises de blé tendre. Sci. Aliments, 3, 181-217.

Bourdet A., Berrier R., Autran J. C., 1972. Critères industriels et critères de sélection pour apprécier la valeur d'utilisation des blés tendres. Ann. Technol. Agric., 21, 163-181.

Cheftel J. C., Cuq J. L., Lorient D., 1981. Protéines alimentaires, 1 vol. Techniques et documentation, Lavoisier, Paris, 309 p.

Chung O. K., Pomeranz Y., 1981. Recent research on wheat lipids. Bakers Dig., 55, 38-50, 55, 96, 97.

Damidaux R., Feillet P., 1978. Relations entre les propriétés viscoélastiques du gluten cuit, la teneur en protéines et la qualité culinaire des blés durs. Ann. Techn. Agric., 27, 799-808.

Feillet P., 1976. Dosage semi-automatique de la teneur en protéines des céréales par méthode Kjeldahl. Tech. Industr. Céréales, 153, 17 19.

Finney K. F., 1985. Experimental breadmaking studies, functional (breadmaking) properties, and related gluten protein fractions. Cereal Foods World, 30, 794-801.

Frazier P. J., Daniels N. W. R., Russel Eggit P. W., 1981. Lipidprotein interactions during dough development. J. Sci. Food Agric., 32, 877-897.

Galliard T., 1986a. Oxygen consumption of aqueous suspensions of wheat wholemeal, bran and germ : involvement of lipase and lipoxygenase. J. Cereal Sci., 4, 33-50.

Galliard T., 1986 $b$. Hydrolytic and oxidative degradation of lipids during storage of wholemeal flour : effects of bran and germ components. J. Cereal Sci., 4, 179-192.
Godon B., 1969. Etude physico-chimique des associations protéiques du gluten de blé. Thèse Faculté des Sciences de Paris, $n^{\circ}$ A.0. 3.274.

Laignelet B., Dumas C., 1984. Oxydations des lipides et distribution des lipides oxydés au cours du pétrissage de la pâte de farine de blé tendre. Lebensm. Wiss. u. Teknol., 17, 226-230.

Mac Master G. J., Bushuk W., 1983. Protein-carbohydrate complexes in gluten: fractionation and proximate composition. $J$. Cereal Sci., 1, 171-184.

Mac Ritchie F., 1981. Flour lipids : theoretical aspects and functional properties. Cereal Chem., 58, 156-158.

Morrison W. R., 1978. Cereal lipids in "Advances in cereal science and technology» Pomeranz Y., ed., AACC Saint Paul, Minnesota, vol. II, 221-348.

Morrison W. R., Mann O. L., Wong S., Coventry A., 1975. Selective extraction and quantitative analysis of non-starch and starch lipids from wheat flour. J. Sci. Food Agric., 26, 507-521.

Morrison W. R., Tan S. L., Hargin K. D., 1980. Methods for the quantitative analysis of lipids in cereal grains and similar tissues. J. Sci. Food Agric., 31, 329-340.

Nguyen-Brem P. T., Kieffer R., Grosch W., 1983. Einfluss von Zusätzen bei der Teigherstellung auf die Molekulargewichte und die Fettsäurezusammensetzung von Kleberfraktionen des Weizens. Getreide Mehl Brot, 37, 35-39.

Ohta S., Torigoe T., Inove S., 1983. Application of enzymatic modification of phospholipids on breadmaking. Cereal Foods World, 28, 561 .

Olcott H. S., Mecham D. K., 1947. Characterization of wheat gluten. I - Protein lipids complex formation during doughing of flours. Lipoprotein nature of the glutenin fraction. Cereal Chem., 24, 407-414.

Pomeranz Y., 1985. Wheat flour lipids, what they can and cannot do in bread. Cereal Foods World, 30, 443-446.

Ponte J. G., De Stephanis V. A., Titcomb S. T., Cotton R. H., 1967. Study of gluten properties as influenced by certain organic solvents. Cereal Chem., 44, 211-220. 